Background As of 15 February 2021, over 20 million people in England have received their first dose of a COVID-19 vaccine. Population-level immunological protection requires the large majority to be vaccinated, though given the spread of misinformation and vaccine hesitancy concerns, this may be challenging. Therefore, this study aimed to elicit from those either undecided or more resistant to having a COVID-19 vaccine, what would make them more likely to have a vaccine when made available to them.

Methods In October/November 2020 we delivered an online population-wide survey of COVID-19 vaccine intention in England. Sampling was stratified by gender, geographical region, and deprivation, with additional purposive sampling of those from an ethnic minority background. An open question 'What might make it more likely that you, your family, or friends would have a coronavirus vaccine when one is ready?' was included. We used content analysis to identify and code emergent themes.

Results We recruited 1660 participants, who did not want (210), had yet to consider (407), or were not sure (1043) whether to be vaccinated. Of these: 946 (57\%) were female, $363(22 \%)$ were of an ethnic minority, and 453 (27\%) lived in the lowest three Index of Multiple Deprivation (small area deprivation statistic) deciles. We received 922 (56\%) open-text responses, with 733 (44\%) detailing at least one factor that would increase their likelihood of receiving a vaccine. The most common concerns expressed was the safety and efficacy of a vaccine, particularly potential side-effects. Participants wanted to see the evidence that supported vaccine approval as many were worried about how quickly vaccines had been developed. Distrust in the Government, specific ministers, and the pharmaceutical companies was a consideration. Some stipulated that it would take mandating for them to have a vaccine. Others wanted it to be free and easy to access. The broader implications of an immunisation programme were of interest, participants wanted to know if the vulnerable would be prioritised and protected and that restrictive conditions would be removed enabling them to engage in a greater range of social activities.

Conclusion Promotional campaigns that focus on COVID-19 vaccine safety and effectiveness, should be prioritised. Provision of accessible comprehendible information that accurately and transparently reports the findings from COVID-19 vaccine trials through a trusted source will be required. Contextualising how vaccines will support the ending of the pandemic and increasing personal and population benefits will be of value.

\section{P104 MITIGATING DIGITAL EXCLUSION IN A REMOTE RESEARCH WORLD: REFLECTIONS FROM CONDUCTING RESEARCH ON POPULATIONS EXPERIENCING HOMELESSNESS DURING THE PANDEMIC}

Emma A Adams*. Population Health Sciences Institute, Newcastle University, Newcastle upon Tyne, UK

\subsection{6/jech-2021-SSMabstracts. 190}

Background The global Covid-19 pandemic challenges researchers to adapt and adjust previous engagement and coproduction strategies in order to continue research projects remotely. Transitioning to remote recruitment and data collection has led to concerns around digital exclusion and further marginalisation of populations that already faced heightened disadvantage. Throughout the pandemic, individuals experiencing homelessness, sometimes perceived as 'harder-to-reach', have faced added challenges for participating in remote research. This study aims to outline critical reflections and observations related to conducting research among individuals experiencing homelessness during the pandemic.

Methods This reflective analysis provides insights from an early career researcher on their experience navigating methodological and logistical challenges that arose while conducting a qualitative study involving individuals experiencing homelessness in the North East of England. Lessons learned on mitigating concerns around digital exclusion were shaped through collaborations with 'Experts by Experience' and analyzing the researcher's reflective journal.

Results Reflections on lessons learned to reduce digital exclusion are centered around three overarching themes: i) meaningful and realistic co-production-ways the research was coproduced with individuals with lived experience; ii) appropriateness as the most important criteria for vouchers and remuneration-process taken to determine the best approaches; iii) inclusiveness and choice for recruitment and data collection strategies to increase participation and reduce participation burden.

Conclusion The responsibility of researchers to find ways to engage homeless populations has become more urgent as the pandemic has created new barriers to access. Concerns about widening the digital divide are given special consideration and approaches used in the context of this study are reviewed and successes are highlighted. Being clear about time and resource expectations while respecting that any commitments are subject to change was helpful for coproduction. Providing participants with physical vouchers required creativity when it came to distribution as gatekeepers were more effective than postal service. Worries about recruitment lag and data collection were overcome by leveraging existing networks and offering participants choice in the way they were contacted and engaged. Conducting research within remote contexts always requires innovation and creativity. However, not all approaches will work for everyone. Guidance on conducting research remotely or while practicing social distancing does not necessarily take into consideration populations facing the greatest marginalisation or digital exclusion. Collectively learning about experiences and approaches to date can ensure that current and future projects do not avoid populations that are likely to experience digital exclusion or other access issues.

\section{P105 PERCEPTIONS AND PRACTICES OF COVID-19 PROTECTIVE BEHAVIORS AMONG THE GENERAL PUBLIC OF NORTH INDIA: FINDINGS OF A QUALITATIVE STUDY POST-LOCKDOWN}

Tuhina Rastogi*, Shally Awasthi, Ranjan Khare, Mahesh Prasad, Gulshan Sami, Vijay Verma. Pediatrics, King George's Medical University, Lucknow, India

\subsection{6/jech-2021-SSMabstracts. 191}

Background India has a high COVID-19 burden. The Indian government responded to the pandemic by mandating its population to adhere to certain Protective Measures (PMs). 
Compliance to these PMs depends on the acceptability and adaptability of the general public to these PMs. However, there is a dearth of studies on this issue. Our study aimed to explore the perceptions and practices of COVID-19 related PMs among the general public in four districts of North India.

Methods The qualitative study was done in four administrative districts (Lucknow, Etawah, Patna and Darbhanga) in North India. All study districts had COVID-19 confirmed active cases at the time of data-collection. Participants in this study were healthy caregivers of under-five children, admitted for pneumonia, in a tertiary care hospital as part of ongoing study. Inclusion criteria was (i) child hospitalized in tertiary care hospital for pneumonia but presently discharged (ii) availability of telephone/mobile with caregiver and (iii) consent to participate. Audio in-depth interviews (IDIs) were conducted. Respondents were purposefully selected to represent both genders and two education groups (i) Below Primary Education Group ( $\leq 5$ years education) and (ii) Above Primary Education Group ( $>5$ years education). This ensured variability and heterogeneity in the population being studied. Data was managed using Atlast Ti. Conventional content analysis was used.

Results From July-Sep 2020, 60 IDIs were conducted; 36.6\% $(22 / 60)$ respondents were females and $26.6 \%(16 / 60)$ had below-primary education. Respondents concurred that most people in their society flouted the recommended PMs. The reasons for poor/non-compliance with PMs were: perceived poor susceptibility to illness, perceived less severity of COVID-19 and low perceived benefits of complying with PMs. Perceived severity of disease played a key role in shaping attitude and practices related to adherence of PMs. Most people believed that COVID-19 often had less severe outcome and is thus less fatal. The lack of direct experience with the disease also attenuated this belief and made them under-estimate the risk due to it. Social stigma associated with the disease caused many to hide the contagion and spread it rapidly. Respondents shared that many people in society 'bide' the disease due to fear of discrimination that was common for COVID-19 positive patients.

In addition, respondents opined that COVID-19 is less prevalent in rural areas and among the less educated population. Most respondents were aware of the recommended PMs and opined that these must be followed, however subjective norms, social norms and behavioural intentions negatively impacted compliance.

Conclusion Since there was poor community perception of susceptibility to COVID-19 as well as poor perceived severity, the community did not understand the benefits of applying to PMs. Therefore, future health communication strategies must take these into account to increase the possibility of success.

\section{P106 'IT'S REALLY STRANGE BECAUSE ALTHOUGH I'M WITH ALL MY FRIENDS, I'VE NEVER FELT SO LONELY AND I HAVE NO IDEA WHY': EXPLORING YOUNG PEOPLE'S EXPERIENCES OF THE COVID-19 PANDEMIC IN NORTH EAST ENGLAND: A QUALITATIVE DIARY-BASED STUDY}

Steph Scott*, Victoria J McGowan, Shelina Visram. Population Health Sciences Institute, Newcastle University, Newcastle-upon-Tyne, UK

10.1136/jech-2021-SSMabstracts. 192
Background Children and young people risk being 'disproportionately harmed' by the COVID-19 pandemic. Whilst an evolving body of literature focuses on the impact of the pandemic on the mental health and wellbeing of children and young people, less attention has been paid to the collection of qualitative, exploratory data. The aim of this study was to examine young people in North East England's experiences of COVID-19 and associated control measures.

Methods Flexible, qualitative diaries were collected with 31 young people aged 13-17 for six weeks between July and October 2020. Diary extracts were curated using Instagram Direct Messaging (DM), email and text messaging. At the end of the study, participants took part in a follow-up interview (conducted by telephone or Zoom), asking them to reflect on their diary entries.

Results Thematic analysis of diaries and interviews yielded three central themes: (1) impacts of social distancing upon mental health and emotional wellbeing; (2) impacts of the pandemic on education and school life; and (3) frustration, burden and responsibility.

Discussion These findings highlight acute mental health impacts (loneliness, isolation, anxiety) as well as longer-term repercussions from disrupted education (missed parts of curriculum, home schooling, cancelled exams, periods of isolation) on young people aged $13-17$ as a result of the COVID-19 pandemic.

\section{P107 WOMEN'S REACTIONS TO THE COVID-19 FOOD SYSTEM SHOCK AND INSIGHTS FOR STRATEGIES SUPPORTING HEALTHY PURCHASING AND DIETARY BEHAVIOURS: A QUALITATIVE STUDY} 1,2Janis Baird, ${ }^{1,2}$ Christina Vogel. 'Medical Research Council Lifecourse Epidemiology Unit, University of Southampton, Southampton, UK; ${ }^{2}$ National Institute for Health Research Biomedical Research Centre, University of Southampton, Southampton, UK; ${ }^{3}$ Global Health Research Institute, School of Human Development and Health, Faculty of Medicine, University of Southampton, Southampton, UK

\subsection{6/jech-2021-SSMabstracts.193}

Background The COVID-19 pandemic disrupted usual routines, including families' food acquisition and consumption patterns. This pandemic could have lasting effects on consumer behaviour and implications for future strategies to improve population diet. The aim of this study was to gain in-depth understanding of families lived experiences by i) examining the impact of disruption resulting from the pandemic on the food purchasing and eating behaviours of young women, and ii) identifying the insights these experiences bring to designing future healthy eating interventions.

Methods A cross-sectional sample of 34 customers aged 1845 years, from a UK discount supermarket chain completed semi-structured telephone interviews. Women were asked questions to understand their lived experiences of food shopping, cooking, and eating during the COVID-19 lockdown. Interviews were transcribed verbatim and analysed thematically using QSR NVIVO software. Six researchers were involved in developing the initial coding framework, double coding of eleven interview transcripts and refining the coding framework. 